\title{
Hallux valgus measurements using weight-bearing computed tomography: what changes?
}

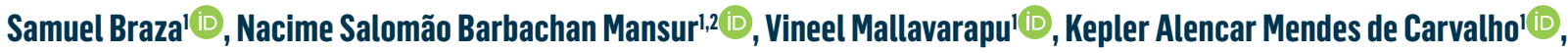 \\ Kevin Dibbern ${ }^{1}$, Caio Augusto de Souza Nery² ${ }^{\circledR}$, Matthieu Laleveéi,3iD, César de César Netto ${ }^{(\mathbb{D}}$ \\ 1. University of Iowa, Carver College of Medicine, Department of Orthopedics and Rehabilitation, Iowa City, IA, USA. \\ 2. Federal University of Sao Paulo, Department of Orthopedics and Traumatology, Sao Paulo, SP, Brazil. \\ 3. Department of Orthopedic Surgery, Rouen University Hospital, Rouen, France.
}

\begin{abstract}
Objective: To assess whether traditional hallux valgus (HV) measurements obtained with conventional radiography (CR) correspond to those obtained with weight-bearing computed tomography (WBCT).

Methods: In this retrospective case-control study, $26 \mathrm{HV}$ feet and 20 control feet were analyzed with CR and WBCT. Hallux valgus angle (HVA), intermetatarsal angle (IMA), interphalangeal angle (IPA), distal metatarsal articular angle (DMAA), sesamoid station (SS), and first metatarsal head shape were measured. Chi-square tests were used to compare hallux valgus and control patients. T-tests were used to compare CR and WBCT. P-values less than 0.05 were considered significant.

Results: WBCT was capable of discriminating patients with HV from controls, showing higher mean values for HV patients than controls in HVA (35.29 and 9.02, p<0.001), IMA (16.01 and 10.01, p<0.001), and DMAA (18.90 and 4.10, p<0.001). When comparing the two methods, differences were not significant between CR and WBCT measurements in HVA $(-0.84, p=0.79)$, IMA $(-0.93, p=0.39)$, IPA (1.53, $p=0.09)$, or SS $(p=0.40)$, but were significant for DMAA $(13.43, p<.0001)$. CR analysis yielded varied metatarsal head shapes, while all WBCT shape classifications were round.

Conclusion: Unidimensional HV measurements were similar between WBCT and CR, while more three-dimensional findings were not. $\mathrm{CR}$ may be used to assess the axial aspects of HV, but multidimensional aspects of the deformity may not be accurately assessed with plain radiographs.
\end{abstract}

Level of Evidence III; Therapeutic Studies; Retrospective Case-Control Study.

Keywords: Hallux valgus; Radiography; Weight-bearing; Computed tomography; Imaging, three-dimensional; Metatarsal bones.

\section{Introduction}

Conventional radiographs $(\mathrm{CR})$ are the standard modality when evaluating patients with hallux valgus $(H V)^{(1,2)}$. In the course of those evaluations, various angular measurements, including the hallux valgus angle (HVA), intermetatarsal angle (IMA), interphalangeal angle (IPA), and distal metatarsal articular angle (DMAA), are obtained(3,4). However, such measurements are sometimes unreliable, and DMAA in particular has less than desired interobserver reliability ${ }^{(4-6)}$. In addition to poor reliability, it is possible these estimates may stray significantly from true values because radiographs only provide images in two dimensions ${ }^{(7)}$.
Many of these radiographic findings and values are used to address possible etiology, grade the deformity, and plan its surgical treatment ${ }^{(8)}$. The shape of the metatarsal head (round, chevron, or flat) and the presence of an abnormal DMAA are associated with the pathogenesis of hallux valgus ${ }^{(9-11)}$. The degree of HVA, IMA, and displacement of sesamoids are used in deformity classifications and treatment algorithms ${ }^{(1,12)}$. Surgical planning is largely based on angular or radiographic unidimensional findings, and deformity recurrence is associated with an inability to correct abnormal angular findings ${ }^{(13)}$. For example, HVA as measured with plain radiographs has been found to be a major predictor of successful hallux valgus correction ${ }^{(14,15)}$.
Study performed at the University of lowa Department of Orthopedics and Rehabilitation, Iowa City, lowa, USA.

Correspondence: César de César Netto. Department of Orthopaedic and Rehabilitation, University of lowa, Carver College of Medicine. 200 Hawkins Dr John PappaJohn Pavillion (JPP). Room 01066, Lower Level. lowa City, IA, 52242 - United States. E-mail: cesar-netto@uiowa.edu. Conflicts of interest: none. Source of funding: none. Date received: November 23, 2021. Date accepted: December 14, 2021. Online: December 20, 2021.
How to cite this article: Braza S, Mansur NSB, Mallavarapu V, Carvalho KAM, Dibbern K, Nery CAS, et al. Hallux valgus measurements using weight-bearing computed tomography: what changes?. J Foot Ankle. 2021;15(3):259-64. 
Weight-bearing computed tomography (WBCT) inherently provides images in three dimensions, and so may offer a more complete way to assess patients with HV and other foot and ankle conditions ${ }^{(16,17)}$. The objective of this study was to compare traditional HV imaging measurements (HVA, IMA, DMMA, head shape, IPA, and sesamoid station [SS]) obtained via CR versus measurements obtained with WBCT. We hypothesized that unidimensional parameters such as HVA, IMA, IPA, and SS would be similar between the two methods, while three-dimensional findings such as DMMA and the shape of the metatarsal head would demonstrate significant differences.

\section{METHODS}

\section{Design}

This study was approved by the University of Iowa's IRB (\#202012422) and complied with the Health Insurance Portability and Accountability Act (HIPAA) and the Declaration of Helsinki. Full weight-bearing radiographs and weight-bearing CTs were taken of HV patients and controls. Patients were enrolled at the University of lowa's Department of Orthopedics and Rehabilitation from January 2017 to November 2020. Included patients were 18 years of age or older with a diagnosis of $\mathrm{HV}$ and matched with control patients. Controls were selected for having HVA $<15^{\circ}$ and no complaints on the forefoot. Patients were excluded from the control group if they had existing foot deformities or any incidental findings of disease on the forefoot.

\section{Subjects}

A total of 19 patients with HV were enrolled (median age 53.79 years, range 18-92, SD 8.09), for a total of 26 feet imaged via radiography and WBCT. The HV patients were $89 \%$ female, $11 \%$ male, and had a median BMI of 29.60 (SD 5.50). The control group was composed of 16 patients (median age 38.18 years, range 17-71, SD 16.37), for a total of 20 imaged feet. Median control BMI was 30.98 (SD 8.29).

\section{Imaging}

Standard bilateral weight-bearing conventional radiographs were taken in anteroposterior, lateral, and oblique views ${ }^{(1)}$. A cone-beam CT extremity scanner (PedCAT; CurveBeam, LLC, Warrington, PA, USA) was used to take weight-bearing CT images of each foot. Each patient was instructed to stand in the scanner with their feet spaced shoulder-width apart, with even distribution of weight ${ }^{(18)}$.

\section{Radiographic Measurements}

Two fellowship-trained, board-certified orthopedic foot and ankle surgeons performed all measurements. HVA, IMA, DMAA, IPA, and sesamoid station (SS) were measured using anteroposterior weight-bearing radiographs (Vue PACS ${ }^{\mathrm{TM}}$, Carestream, USA). SS was measured between the medial sesamoid and the metatarsal axis using the Hardy-Clapham system $^{(19)}$. Metatarsal head shape was classified through observation into 3 categories: flat, round, and chevron ${ }^{(9)}$.

\section{WBCT measurements}

The same two fellowship-trained foot and ankle surgeons performed all WBCT measurements. CubeVue ${ }^{\mathrm{TM}}$ software (CurveBeam, LLC, Warrington, PA, USA), was used to analyze the images, which were converted from raw multiplanar data into sagittal, coronal, and axial plane images. Head shape, SS, HVA,

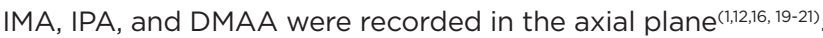

The metatarsal and phalanx axes were established in the axial plane, and angular measurements were performed using the Cobb method ${ }^{(16,22)}$. The most medial and the most lateral articular voxel of the metatarsal head were used to establish the DDMA ${ }^{(23)}$ (Figure 1).

\section{Statistical Analysis}

Interobserver reliability was calculated with intraclass correlation coefficients (ICCS) for continuous data and Cohen's kappa for categorical data. Descriptive statistics including mean, median, range, interquartile range (IQR), standard deviation and error, and 95\% confidence intervals were calculated for each measurement. The Shapiro-Wilk test was used to evaluate normality. One-way ANOVA was used to compare the means of normally distributed variables, while nonparametric Wilcoxon tests were used for non-normally distributed variables. T-tests were used to compare measurements between CR and WBCT. Chi-square tests were used to evaluate differences between $\mathrm{HV}$ and control patients. P-values less than 0.05 were considered significant.

\section{Results}

Interobserver reliability was generally high. The ICC was 0.91 for HVA measured via CR and 0.98 for HVA measured via WBCT. ICC Was 0.89 for IMA as measured by both radiograph and WBCT. For DMAA, the ICC was 0.83 and 0.72 using CR and WBCT, respectively. Additionally, ICC for IPA was 0.80 as measured by plain radiography and 0.77 as measured by WBCT. For sesamoid station, Cohen's kappa was calculated to be 0.70 with CR and 0.75 with WBCT (Figure 2).

Using WBCT scans, readers were able to accurately discern $\mathrm{HV}$ patients from controls without HV (Figure 3). Using WBCT, the mean HVA in HV patients was $35.29^{\circ}$ compared to $9.02^{\circ}$ for control patients $(p<0.001)$. IMA was also significantly greater in $\mathrm{HV}$ patients compared to controls (mean $16.01^{\circ}$ and $10.01^{\circ}$ respectively, $\mathrm{p}<0.001$ ). A significant difference was detected for IPA (mean $5.87^{\circ}$ for $\mathrm{HV}$ patients and $11.19^{\circ}$ for controls, $p<0.001)$. DMAA was found to be significantly greater in $\mathrm{HV}$ patients than controls (mean $18.90^{\circ}$ vs $4.10^{\circ}$, $\mathrm{p}<0.001)$. SS was also significantly different in $\mathrm{HV}$ patients compared to controls ( $p<0.001)$.

Comparing $\mathrm{CR}$ and $\mathrm{WBCT}$, some angles were similar and others were significantly different (Figure 4). For pooled HV and control patients, similarities were seen between HVA measured by radiography and HVA measured by WBCT (mean difference $=-0.84, p=0.79, C L=-6.40,4.73$ ). For pooled $\mathrm{HV}$ and control patients, similarities were also seen in IMA 

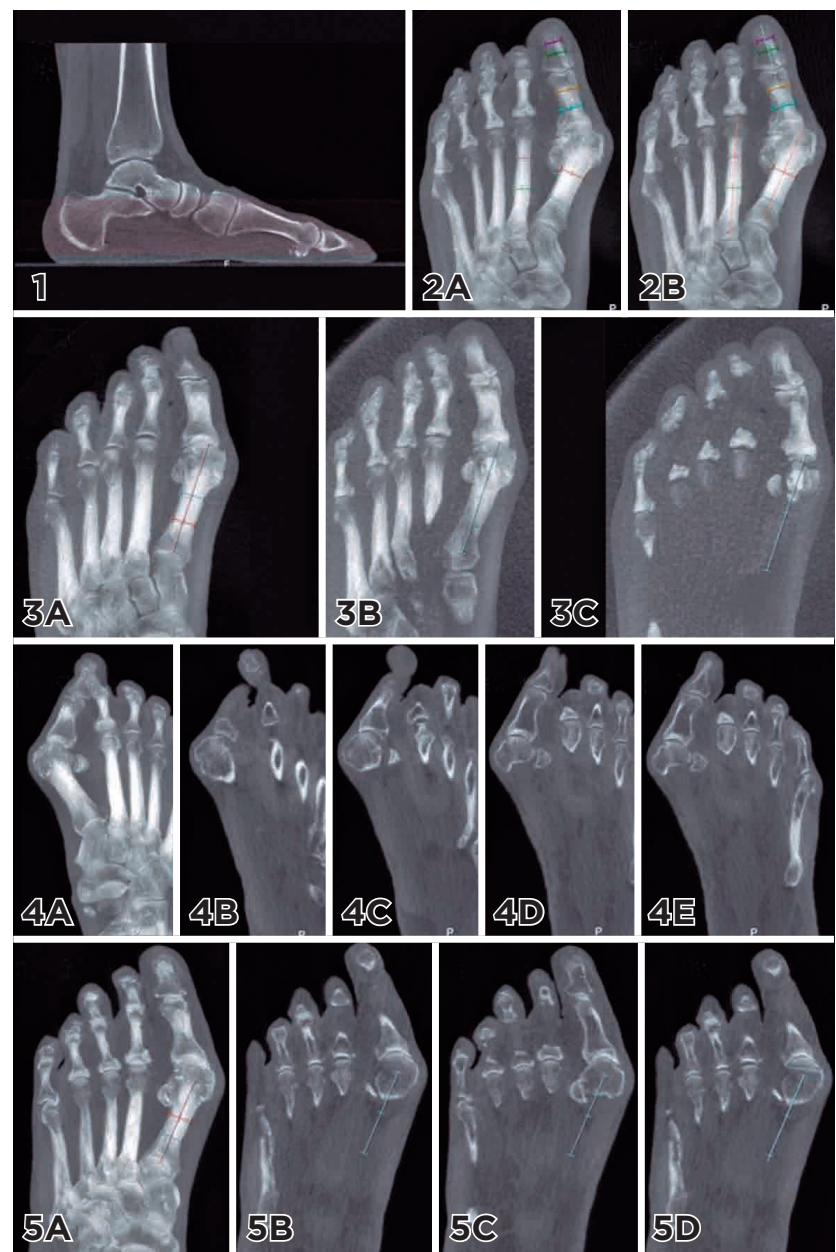

Figure 1. Sequence of hallux valgus angles measurements using weight-bearing computed tomography (WBCT). Panel 1 demonstrates the increase (approximately $29.5 \mathrm{~mm}$ ) of the slab (cut thickness) used to find bone axes when the visualization is changed to the axial window. Panel 2 shows measurements of traditional hallux valgus angles: intermetatarsal angle (IMA), hallux valgus angle (HVA), and interphalangeal angle (IPA). The thicker slab is used to find the metatarsal and phalanxes (2A) axes, followed by angular calculations (2B). Hardy and Clapham assessment on WBCT is shown on panel 3, where a thicker slab is used the find the first metatarsal axis ( $3 A$ ), and the exact point where this axis crosses the medial sesamoid is observed by moving inferiorly (3B), portraying the amount of dislocation presented (3C). Panel 4 illustrates how the first metatarsal head shape is evaluated under WBCT, decreasing the slab (4A to $4 \mathrm{~B}$ ) to its standard cut thickness $(0.37 \mathrm{~mm})$ and observing the entire head diameter from dorsal (4B $\mid 4 C$ ) to plantar (4D $4 \mathrm{E}$ ). After finding the first metatarsal axis $(5 A)$ with the thicker slab, the cut is reduced to its standard value. The distal metatarsal articular angle (DMAA) is obtained, as shown in panel 5. The most medial (5B) and lateral (5C) articular points of the first metatarsal head are attained using all WBCT axial cuts. The complementary angle between the line connecting these two points and the first metatarsal axis represents the DMAA (5D).


Figure 2. Example of the measured variables in a hallux valgus patient using conventional weight-bearing radiographs and weightbearing computed tomography (WBCT). Traditional hallux valgus angles, such as hallux valgus angle (HVA), intermetatarsal angle (IMA) and interphalangeal angle (IPA) assessed on radiographs (A) and WBCT (B). Shape of the first metatarsal head on radiographic (C) and tomographic (D) analysis. Measurement of the distal metatarsal articular angle (DMAA) using radiographs (E) and WBCT (F). Finally, the sesamoid station calculated under a radiographic view (G) and a weight-bearing computed tomography view $(H)$.

(mean difference $=-0.93, \mathrm{p}=0.39, \mathrm{CL}=-2.89,1.02$ ) and IPA (mean difference $=1.53, \mathrm{p}=0.09, \mathrm{CL}=-0.48,3.53$ ). $\mathrm{SS}$ was also similar when using WBCT in comparison to $C R(p=0.40)$. However, DMAA measurements were found to be significantly different in the pooled patient population between WBCT and CR (score mean difference $=13.43, \mathrm{p}<.0001$, $C L=9.00,18.30$ ).

For HV patients, $65.4 \%$ of metatarsal head shapes were classified as round and $34.6 \%$ as chevron. For control patients, $50 \%$ of metatarsal head shapes were classified as round, $45 \%$ were classified as chevron, and $5 \%$ were classified as flat. In contrast, for both patient populations $100 \%$ of WBCT classifications of metatarsal head shape were round (Figure 3 ). Correlation analysis ( $r$ ) was also performed between angles measured by radiograph and angles by WBCT. The coefficients were $r=0.97$ for HVA, $r=0.88$ for IMA, $r=0.51$ for IPA, and $r=0.61$ for DMAA. 

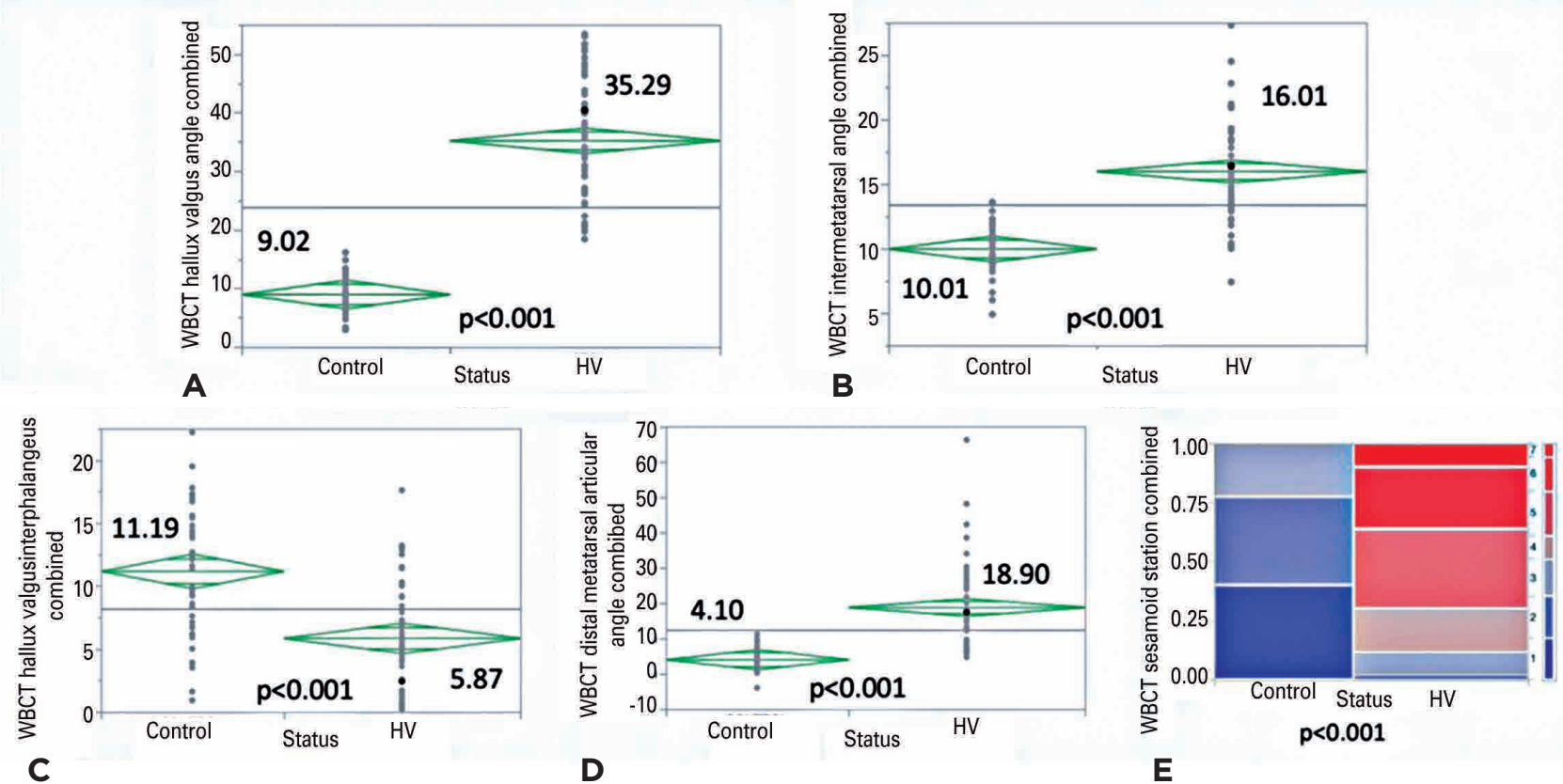

Figure 3. Comparison of hallux valgus patients and control patients using measurements from weight-bearing computed tomography (WBCT). Mean values for each measurement are given. A) Hallux valgus angle (HVA), B) intermetatarsal angle (IMA), C) hallux valgus interphalangeous angle (HVIP or IPA), D) distal metatarsal articular angle (DMAA), and E) sesamoid station (SS).

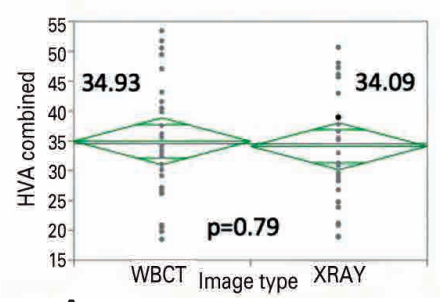

A

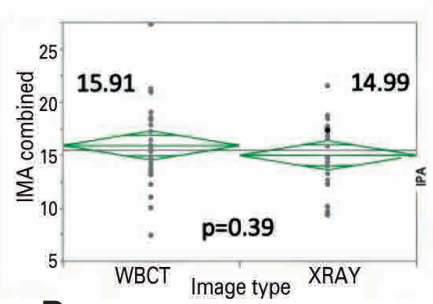

B

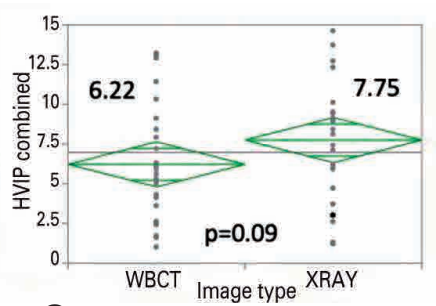

C

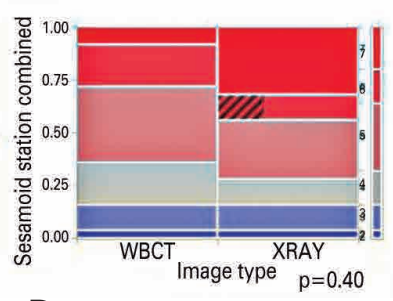

D


Figure 4. Comparison of hallux valgus measurements obtained from weight-bearing computed tomography (WBCT) and conventional radiography $(C R)$ for the combined hallux valgus and control patient population. Mean values for each measurement are given. $A$ ) Hallux valgus angle (HVA), B) intermetatarsal angle (IMA), C) hallux valgus interphalangeal angle (HVIP or IPA), D) sesamoid station (SS), E) distal metatarsal articular angle, F) metatarsal head shape measured by CR, and G) metatarsal head shape measured by WBCT.

\section{Discussion}

Hallux valgus is one of the most common problems seen and addressed by foot and ankle surgeons. HVA, IMA, and a number of other important metrics are used in the diagnosis, surgical planning, and postoperative analysis of $\mathrm{HV}$, and so accurate measurement of these parameters is important. Therefore, this retrospective study aimed to compare such parameters as measured by conventional weight-bearing radiographs with those obtained with weight-bearing computed tomography (WBCT). 
Reliability was good for both radiography and WBCT across the overall patient population enrolled in the study. WBCT was found to be more reliable than $\mathrm{CR}$ between observers for HVA and SS, equivalent in reliability for IMA, and less reliable for DMAA and IPA. Since many clinicians may not have access to WBCT, the solid reliability of CR for most measurements is helpful for the assessment and treatment of HV. Considering that WBCT and CR operate in different dimensions, it is unsurprising that reliabilities varied depending on the specific measurement.

As was hypothesized, for some parameters (HVA, IMA, IPA, and SS), radiographic measurements were comparable to WBCT. This aligned with the findings of Mahmoud et al. ${ }^{(16)} \mathrm{Ba}-$ sed on this study's findings, and the fact that these measurements assess a unidimensional aspect of the deformity, there should be a high degree of comparability between $C R$ and WBCT measurements. It appears that the two methods can be used interchangeably when evaluating HVA, IMA, IPA, and SS.

However, there was a statistically significant difference between the radiographically derived measurements of DMAA and those obtained from WBCT. This may be explained by the more three-dimensional nature of the DMAA angle, as well as the fact that DMAA varies with first metatarsal axial rotation and inclination ${ }^{(8)}$. Given the observed discrepancy, use of DMAA in clinical practice may necessitate WBCT imaging rather than $\mathrm{CR}$. Similarly, there was a stark difference between metatarsal head shape as measured with radiography versus WBCT. When measured with radiography, there were a variety of head shape classifications, yet when using WBCT all head shapes were found to be round across both $\mathrm{HV}$ patients and controls. WBCT shows the true shape of the metatarsal head more than radiography does, and so this finding implies that head shape may not be a strong etiological factor, in contrast to what some authors have indicated in the past $^{(9-11)}$. DMAA and head shape are three-dimensional parameters, and as a result may be greatly influenced by factors like the position of the foot, the inclination of the metatarsal, and rotation.

This study has several limitations that must be addressed. First, it was a retrospective analysis, and is thus subject to inherent bias. Functional evaluation was not performed, which hinders the possibility of associating clinical symptoms and radiographic aspects of hallux valgus. Proximal features of the deformity at the tarsometatarsal and naviculocuneiform joints were not tested. Angles assessing the rotation of the metatarsal or the sesamoids were not considered in this study, since the inability of radiographs to estimate true values is already known $^{(24,25)}$. Although healthy controls with an absence of foot deformities were included, they were not volunteers. Further, although statistical differences were found, no sample size calculation or power analysis was performed.

\section{Conclusion}

HV values obtained with WBCT had good reliability. Indirect unidimensional radiographic measurements of HVA and IMA are not significantly different from HVA and IMA measurements obtained with WBCT. This may support the use of $\mathrm{CR}$ to address the disease's axial components. However, the measurement of DMAA changed significantly between radiographs and WBCT, which might pose a challenge to providers when assessing this aspect of the deformity.

Authors' contributions: Each author contributed individually and significantly to the development of this article: SB *(https://orcid.org/0000-0001-83811455) Interpreted the results of the study, bibliographic review, wrote the paper, formatting of the article, participated in the reviewing process, approved the final version; NSBM *(https://orcid.org/0000-0003-1067-727X) Conceived and planned the activities that led to study, participated in the review process, approved the final version; VM *(https://orcid.org/0000-0002-8612-5941) Participated in the review process, approved the final version; KAMC *(https://orcid.org/0000-0003-1082-6490) Participated in the review process, approved the final version; KD *(https://orcid.org/0000-0001-5404-2132) Bibliographic review, participated in the review process, approved the final version; CASN *(https://orcid.org/0000-0002-9286-1750) Bibliographic review, approved the final version; $\mathrm{ML}{ }^{*}$ (https://orcid.org/0000-0001-5058-8867) Participated in the review process, approved the final version; CCN *(https:// orcid.org/0000-0002-9286-1750) Conceived and planned the activities that led to the study, performed the surgeries, approved the final version. Al authors read and approved the final manuscript. * ORCID (Open Researcher and Contributor ID) (iD).

\section{References}

1. Coughlin MJ, Jones CP. Hallux valgus: demographics, etiology, and radiographic assessment. Foot Ankle Int. 2007;28(7):759-77.

2. Coughlin MJ, Kaz A. Correlation of Harris mats, physical exam, pictures, and radiographic measurements in adult flatfoot deformity. Foot Ankle Int. 2009;30(7):604-12.

3. Coughlin MJ, Freund $\mathrm{E}$. The reliability of angular measurements in hallux valgus deformities. Foot Ankle Int. 2001;22(5):369-79.

4. Lee KM, Ahn S, Chung CY, Sung KH, Park MS. Reliability and relationship of radiographic measurements in hallux valgus. Clin Orthop Relat Res 2012;470(9):2613-21.
5. Vittetoe DA, Saltzman CL, Krieg JC, Brown TD. Validity and reliability of the first distal metatarsal articular angle. Foot Ankle Int. 1994;15(10):541-7.

6. Chi TD, Davitt J, Younger A, Holt S, Sangeorzan BJ. Intra-and inter-observer reliability of the distal metatarsal articular angle in adult hallux valgus. Foot Ankle Int. 2002;23(8):722-6.

7. Robinson AH, Cullen NP, Chhaya NC, Sri-Ram K, Lynch A. Variation of the distal metatarsal articular angle with axial rotation and inclination of the first metatarsal. Foot Ankle Int. 2006;27(12):1036-40. 
8. Perera AM, Mason L, Stephens MM. The pathogenesis of hallux valgus. J Bone Joint Surg Am 2011;93(17):1650-61.

9. Ferrari J, Malone-Lee J. The shape of the metatarsal head as a cause of hallux abductovalgus. Foot Ankle Int. 2002;23(3):236-42.

10. Piggott $\mathrm{H}$. The natural history of hallux valgus in adolescence and early adult life. J Bone Joint Surg Br 1960;42(4):749-60.

11. van Deventer SJ, Strydom A, Saragas NP, Ferrao PNF. Morphology of the first metatarsal head as a risk factor for hallux valgus interphalangeus. Foot Ankle Surg. 2020;26(1):105-9.

12. Shi GG, Whalen JL, Turner NS 3rd, Kitaoka HB. Operative Approach to Adult Hallux Valgus Deformity: Principles and Techniques. J Am Acad Orthop Surg. 2020;28(10):410-8.

13. Hagio T, Yoshimura I, Kanazawa K, Minokawa S, Ishimatsu T, Nagatomo $M$, et al. Risk factors for recurrence of hallux valgus deformity after minimally invasive distal linear metatarsal osteotomy. J Orthop Sci. 2021:S0949-2658(20)30379-1.

14. Deenik AR, de Visser E, Louwerens JW, de Waal Malefijt M, Draijer FF, de Bie RA. Hallux valgus angle as main predictor for correction of hallux valgus. BMC Musculoskelet Disord. 2008;9(1):1-6.

15. Okuda R, Kinoshita M, Yasuda T, Jotoku T, Shima H, Takamura M. Hallux valgus angle as a predictor of recurrence following proximal metatarsal osteotomy. J Orthop Sci. 2011;16(6):760-4.

16. Mahmoud K, Metikala S, Mehta SD, Fryhofer GW, Farber DC, Prat D. The role of weightbearing computed tomography scan in hallux valgus. Foot Ankle Int. 2021;42(3):287-93.

17. Lalevée M, Barbachan Mansur NS, Lee HY, Maly CJ, lehl CJ, Nery C, Lintz F, de Cesar Netto C. Distal Metatarsal Articular Angle in Hallux Valgus Deformity. Fact or Fiction? A 3-Dimensional Weightbearing CT Assessment. Foot Ankle Int. 2021; 13:10711007211051642.
18. Mansur NSB, Lalevee M, Schmidt E, Dibbern K, Wagner P, Wagner $\mathrm{E}$, et al. Correlation between indirect radiographic parameters of first metatarsal rotation in hallux valgus and values on weightbearing computed tomography. Int Orthop. 2021;45(12):3111-8.

19. Hardy RH, Clapham JC. Observations on hallux valgus; based on a controlled series. J Bone Joint Surg Br. 1951;33-B(3):376-91.

20. Wagner P, Wagner E. Is the Rotational Deformity Important in Our Decision-Making Process for Correction of Hallux Valgus Deformity? Foot Ankle Clin. 2018;23(2):205-17.

21. Coughlin MJ, Saltzman CL, Nunley JA 2nd. Angular measurements in the evaluation of hallux valgus deformities: a report of the ad hoc committee of the American Orthopaedic Foot \& Ankle Society on angular measurements. Foot Ankle Int. 2002;23(1):68-74.

22. Apostle KL, Coleman NW, Sangeorzan BJ. Subtalar joint axis in patients with symptomatic peritalar subluxation compared to normal controls. Foot Ankle Int. 2014;35(11):1153-8.

23. Day J, de Cesar Netto C, Richter M, Mansur NS, Fernando C, Deland JT, et al. Evaluation of a Weightbearing CT Artificial IntelligenceBased Automatic Measurement for the M1-M2 Intermetatarsal Angle in Hallux Valgus. Foot Ankle Int. 2021;42(11):1502-9.

24. Sadamasu A, Yamaguchi S, Kimura S, Ono Y, Sato Y, Akagi R, et al. Influence of foot position on the measurement of first metatarsal axial rotation using the first metatarsal axial radiographs. J Orthop Sci. 2020;25(4):664-70.

25. Yildirim Y, Cabukoglu C, Erol B, Esemenli T. Effect of metatarsophalangeal joint position on the reliability of the tangential sesamoid view in determining sesamoid position. Foot Ankle Int. 2005;26(3):247-50. 